\title{
Cytogenetical Evidence for Latent Centromeres and Reactivation in Chromosomes of Haplopappus gracilis (Asteraceae)
}

\author{
Hidehiro Hanmoto ${ }^{1, *}$, Yoshihiko Yonezawa ${ }^{2}$ and Katsuhiko Kondo ${ }^{3}$ \\ ${ }^{1}$ Ohmiya High School, Amanuma, Saitama, 330-0834, Japan \\ ${ }^{2}$ Department of Biology, Naruto University of Education, Naruto, 772-8502, Japan \\ ${ }^{3}$ Laboratory of Plant Chromosome and Gene Stock, Graduate School of Science, \\ Hiroshima University, Kagamiyama, Higashi-Hiroshima, 739-8526, Japan
}

Received September 18, 2007; accepted December 1, 2007

\begin{abstract}
Summary Rearranged karyotypes of $2 n=5$ present in three plants of Haplopappus gracilis $(2 n=4)$ were cytogenetically analyzed to clarify the origin of an additional centromere in the chromosome complement. One of the plants was a progeny of the homozygote with normal chromosome 1 crossed with the heterozygote with normal and centromere-shifted chromosome 1 . The other two plants were derived from seedlings irradiated with X-rays during early germination stage. The chromosome complement of $2 n=5$ found in the first plant was comprised of 3 normal chromosomes (one chromosome 1 and two chromosome 2) and two rearranged chromosomes each possessing a centromere at the subterminal position. All the chromosome complements of the irradiated plants were similar to each other and each consisted of three normal chromosomes (one chromosome 1 and two chromosome 2) and 2 rearranged chromosomes, one large and metacentric and the other, fragmentlike. The origin of the additional centromere in each complement of $2 n=5$ is discussed from the point of chromosomal evolution in the genus Haplopappus.
\end{abstract}

Key words Chromosomal evolution, Haplopappus gracilis, Latent centromere, Reactivation

Haplopappus gracilis (Nutt.) Gray, an annual species of the Asteraceae, shows the lowest chromosome number of $n=2$ in higher plants (Jackson 1957). The chromosome complement of this species consists of a larger metacentric chromosome pair (chromosome A or chromosome 1) and a shorter subtelocentric chromosome pair with a satellite (chromosome B or chromosome 2) (Jackson 1959, Tanaka 1967). The chromosome complement of $n=2$ in this species is considered to be reconstituted from that of $n=4$ in $H$. ravenii, an allied species, by chromosome rearrangement (Jackson 1962, Tanaka 1967, Ikeda 1987). Assuming this to be so, discussion is made in the following of the whereabouts of 2 additional centromeres present in the chromosome complement of $H$. ravenii.

Based on analysis of the chromosome configuration of $\mathrm{F}_{1}$ hybrids between $\mathrm{H}$. gracilis and $H$. ravenii, Jackson (1962) maintains the chromosome segments of both species to be almost entirely homologous. His study assumes the chromosome complement of $\mathrm{H}$. gracilis to derive from that of $H$. ravenii through a series of unequal reciprocal translocations, causing the extra centromeres to be lost as B chromosomes. Based on comparative analysis of mitotic chromosomes at prophase and metaphase in either species, Tanaka (1967) also considers that the chromosomes of $H$. gracilis have evolved from those of $H$. ravenii via chromosome breakage and end-to-end tandem fusion. His study makes no comment on the sites of extra centromeres of $H$. ravenii.

In the centric transposition race of $H$. gracilis, or Mexican race, Jackson (1973) has shown the centromere in chromosome 1 (or chromosome A) to be situated at a subterminal position and he

*Corresponding author, e-mail: hanmot@nifty.com 
maintains based on the pachytene configuration of $F_{1}$ hybrid between the standard race and the Mexican race, the transposition of centromere in this chromosome to arise from a three-break phenomenon, a chromosomal break on both sides of the centromere and a third break at the subterminal position of one arm.

Yonezawa (1981a) has also noted a similar centromere-shifted chromosome 1 in several plants from a reserved population of this species. This reserved population has been originated from about 20 achenes collected by Jackson ( $c f$. Tanaka 1967). But Yonezawa (1981a) considers transposition of the centromere to come about through the disappearance of a centromere situated at the median position and the appearance of a subterminal position in the long arm of chromosome 1 , and the centromere is transposed from the subterminal position. The appearance of new centromere may be considered due to reactivation of the centromere suppressed during the course of chromosomal evolution from $H$. ravenii to $H$. gracilis.

We previously reported the karyotypes of $2 n=5$ observed in the plants irradiated by X-rays and pointed out that the extra centromere in the $2 n=5$ complement might be derived from the reactivation of the suppressed centromere in the chromosome 1 in H. gracilis (Hanmoto et al. 2003).

The present study directs careful attention to the sites of extra centromeres present in the chromosome complement of $H$. ravenii during chromosomal evolution in this genus, based on cytogenetical analysis of 1 plant with $2 n=5$ observed in these 2 plants with $2 n=5$ artificially induced by $\mathrm{X}$-ray irradiation, and in artificially pollinated progenies of the homozygote with normal chromosome 1 crossed with the heterozygote possessing normal and centromere-shifted chromosome 1.

\section{Materials and methods}

\section{Plant material}

Three chromosomal mutants with $2 n=5$ in Haplopappus gracilis (Nutt.) Gray, an annual species of the Asteraceae, were used: One mutant was a progeny of the homozygote of normal chromosome 1 crossed with the heterozygote of normal and centromere-shifted chromosome 1 (Yonezawa 1981b). Out of 160 progenies, only one plant (designated as 78-465) possessed $2 n=5$ chromosomes. The other two plant materials, X18-38 and X18-49, were found growing among 133 plants from the achenes irradiated by X-rays just at the time of germination and had $2 n=5$ with a fragment-like chromosome (Hanmoto et al. 2003).

These three mutants were derived from the reserved, Kansas-Hiroshima Strain No. 1 (KH-1), of Haplopappus gracilis, which was established by Prof. Tanaka in 1967 and presently is being kept at the Laboratory of Plant Chromosome and Gene Stock of Hiroshima University until this time. The morphological and cytological characteristics of this strain are presented in the paper submitted by Tanaka (1967).

\section{Chromosome preparation and karyotype analysis}

The preparation was the same as previously reported (Yonezawa 1981a, Hanmoto et al. 2003): Root tips were pretreated with $0.002 \mathrm{M} 8$-hydroxyquinoline solution for $90 \mathrm{~min}$ at $18^{\circ} \mathrm{C}$ or $0.1 \%$ colchicine solution for $60 \mathrm{~min}$ at $20^{\circ} \mathrm{C}$ followed by fixation in $45 \%$ acetic acid solution for $15 \mathrm{~min}$ at $5^{\circ} \mathrm{C}$. The tips were then macerated in a mixture of $1 \mathrm{~N} \mathrm{HCl}$ and $45 \%$ acetic acid $(2: 1)$ for $15 \mathrm{~s}$ at $60^{\circ} \mathrm{C}$. The terminal $1-2 \mathrm{~mm}$ root portions were cut off and stained with 1 or $2 \%$ aceto-orcein solution and then squashed under cover slips.

The well-spread prophase and metaphase chromosomes were microscopically analyzed for assessment of the chromosome size, centromere position and distribution of early condensing regions (ECRs).

For examination of meiotic chromosomes in pollen mother cells (PMCs), the immature heads were fixed in a ethanol, chloroform and acetic acid $(2: 1: 1)$ mixture for over $1 \mathrm{~h}$ and stained with 1 
or $2 \%$ aceto-orcein solution, or Feulgen reaction followed by $1 \%$ aceto-orcein solution.

\section{Results}

\section{Standard karyotype of $H$. gracilis}

Figs. 1A and B indicate the standard karyotype of the KH-1 of $\mathrm{H}$. gracilis to be comprised of a longer metacentric pair (chromosome A by Jackson (1962) or chromosome 1 by Tanaka (1967)) and a shorter subtelocentric pair with satellite (chromosome B or chromosome 2), which were designated as $1 \mathrm{~g}$ and $2 \mathrm{~g}$, respectively, according to the criteria of Tanaka (1967), so as to avoid confusing chromosome B with B chromosome, meaning a supernumerary chromosome. By this designation, normal karyotype of the KH-1 of $H$. garacilis may be expressed as $2 n=4=1 \mathrm{~g}+1 \mathrm{~g}+2 \mathrm{~g}+2 \mathrm{~g}$. The features of this karyotype are consistent with these of the original strain of this species as previously described by Jackson (1962).

In the mitotic prophase, $1 \mathrm{~g}$ showed three early condensing regions (ECRs) in the centromeric region and interstitial and subdistal regions of the long arm. There were one ECR containing the centromeric region and a whole short arm in $2 \mathrm{~g}$ (Fig. 1A). These findings are consistent with and confirm data in previous papers (Tanaka 1967, Yonezawa 1981a, Ikeda 1987, Hanmoto et al. 2003).

Fig. $1 \mathrm{C}$ shows two bivalent chromosomes of $1 \mathrm{~g}-1 \mathrm{~g}$ and $2 \mathrm{~g}-2 \mathrm{~g}$ at first metaphase in PMCs with regular configuration.

\section{Rearranged karyotype with $2 n=5$}

Figs. 1D-I show rearranged karyotypes each comprised of five chromosomes at mitotic and meiotic stages observed in the 78-465, X18-38 and X18-49 plants. Three rearranged karyotypes with the karyotype formula heading are described and discussed in the following.

(1) 78-465 $\left(2 n=5=1 \mathrm{~g}+2 \mathrm{~g}+2 \mathrm{~g}+\right.$ two rearranged chromosomes, i.e., $\mathrm{V}_{1}$ and $\mathrm{V}_{2}$. Figs. 1D-F)

The larger rearranged chromosome, $\mathrm{V}_{1}$, had a centromere at the subterminal position and one ECR at the centromeric position. The smallest one, $\mathrm{V}_{2}$, also had a subterminal centromere, but also 2 ECRs at the centromeric and subdistal positions of the long arm (Figs. 1D and E). In the diakinesis of PMCs, $V_{1}$ and $V_{2}$ were seen to be paired with $1 \mathrm{~g}$ (Fig. $1 \mathrm{~F}$ ), but in some cases, the univalent of $\mathrm{V}_{2}$ was also present (data not shown).

(2) X18-38 $\left(2 n=5=1 \mathrm{~g}+2 \mathrm{~g}+2 \mathrm{~g}+\right.$ two rearranged chromosomes, i.e., $\mathrm{V}_{3}$ and "F". Figs. $1 \mathrm{G}$ and $\mathrm{H}$ )

The karyotype of this plant has been described preliminary in our previous report, except for the designation of $\mathrm{V}_{3}$ and "F" (Hanmoto et al. 2003).

The larger rearranged chromosome of $\mathrm{V}_{3}$ was metacentric and shorter than normal $1 \mathrm{~g}$. "F" was the smallest chromosome with a median centromere in the chromosome complement (Fig. 1G). During mitotic prophase, the $\mathrm{V}_{3}$ chromosome was seen to have 2 ECRs at the centromeric position as well as the subdistal position of the short arm. "F" was comprised of one ECR which occupied nearly the entire region of the chromosome (data not shown, cf. Hanmoto et al. 2003). The segregation of this chromosome at anaphase was regular (data not shown), indicating it to possess an active centromere. During the diakinesis and the first metaphase of PMCs, the $\mathrm{V}_{3}$ chromosome became paired with $1 \mathrm{~g}$, though "F" was always univalent in the vicinity of $1 \mathrm{~g}-\mathrm{V}_{3}$ bivalent (Fig. $\left.1 \mathrm{H}\right)$.

(3) X18-49 $\left(2 n=5=1 \mathrm{~g}+2 \mathrm{~g}+2 \mathrm{~g}+\right.$ two rearranged chromosomes, i.e., $\mathrm{V}_{3}$ and "F". Fig. 1I)

In mitosis in 4 of the eight roots investigated, a karyotype with $2 n=5$ similar to that of X18-38 was observed (Fig. 1I). The karyotypes of the other roots were normal, thus demonstrating normal and rearranged karyotypes in root system in this plant. This rearranged karyotype was also reported in our previous report (Hanmoto et al. 2003).

The first metaphase in PMCs showed 2 regular bivalents in all cases, as with plants having the normal karyotype of $2 n=4$ (data not shown). The rearranged karyotype in this plant may thus be 


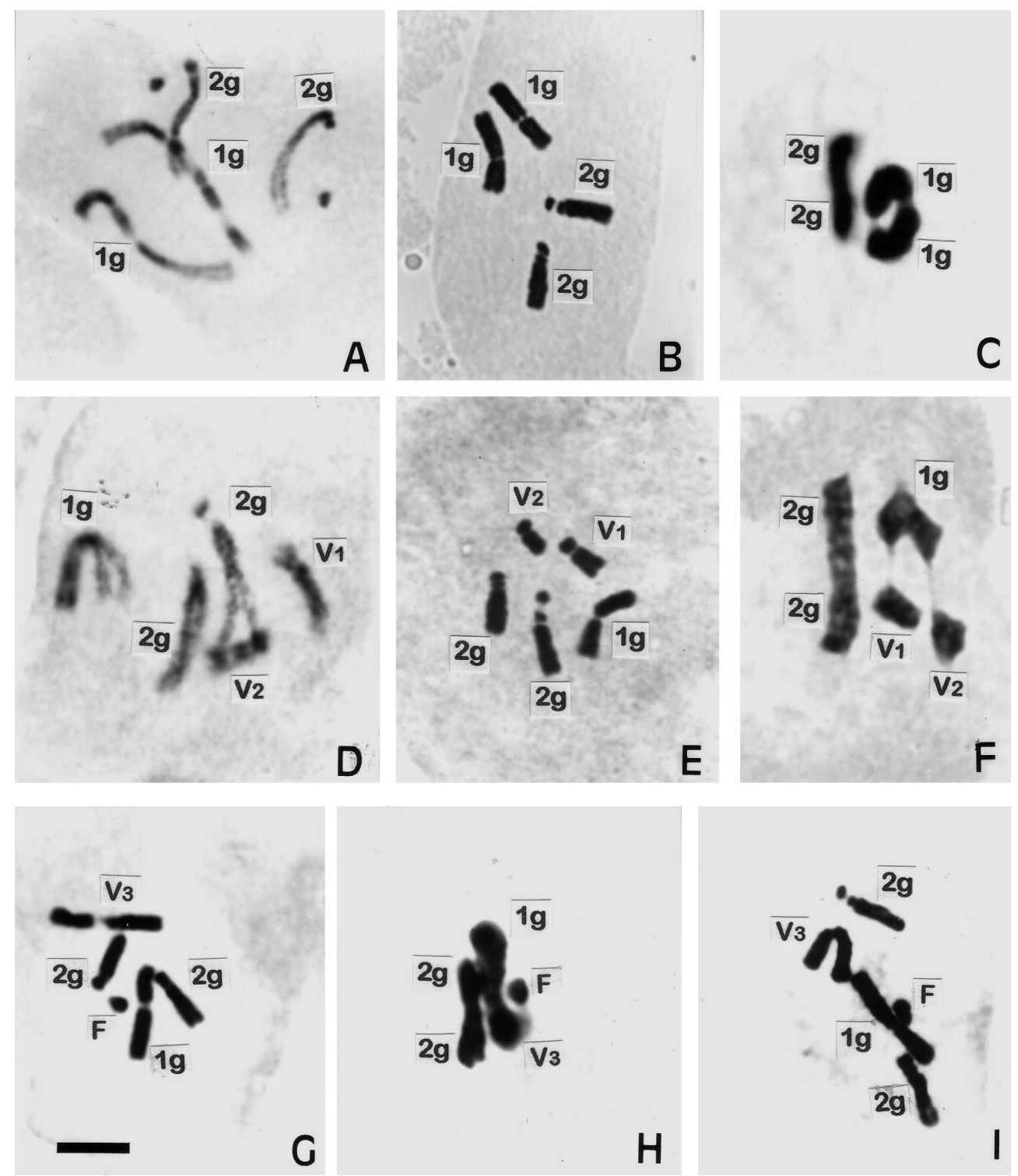

Fig. 1. Photomicrographs of mitotic prophases (A and D) and metaphases (B, E, G and I) and first meiotic metaphases $(\mathrm{C}, \mathrm{F}$ and $\mathrm{H})$ of the normal karyotype with $2 n=4(\mathrm{~A}-\mathrm{C})$ and the rearranged karyotype of 2n=5, 78-465 (D-F), X18-38 (G and H) and X18-49 (I) in H. garacilis. Refer to results for explanation of letters appearing on chromosomes. Scale bar in G, $5 \mu \mathrm{m}$.

considered to induce only in the roots by X-rays irradiation.

\section{Discussion}

Chromosomes possessing 2 or more centromeres (dicentrics or multicentrics) produced by chromosomal rearrangement such as reciprocal translocation, are usually eliminated from cell populations during mitosis owing to the irregular distribution of such chromosomes. The continued existence of dicentrics or multicentrics would thus require the elimination of 1 or more centromeres 
ravenii

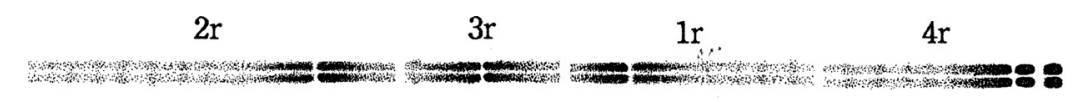

gracilis

$1 \mathrm{~g}$

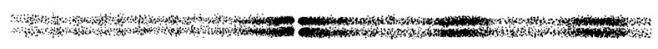

$\mathrm{V} 1$

78-465

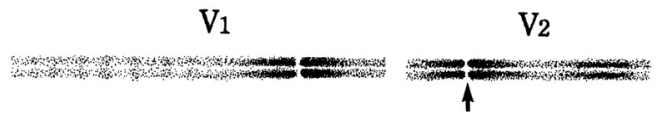

$\mathrm{V} 3$
$2 \mathrm{~g}$

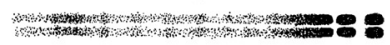

$2 \mathrm{~g}$

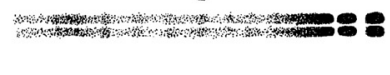

$2 \mathrm{~g}$

$\mathrm{X} 18-38 \& 49$
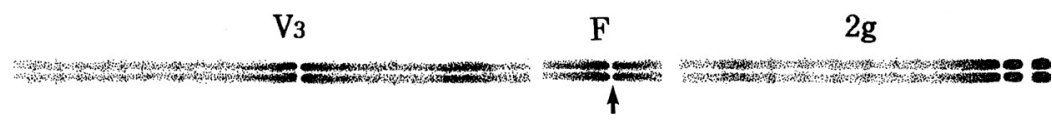

Fig. 2. Semi-schematic representation of haploid chromosome complement at mitotic prophases of Haplopappus ravenii, $H$. garacilis, 78-465, X18-38 and X18-49. Chromosome arrangement and agreement between ravenii and gracilis are in accordance with of Ikeda (1987). The distribution of ECRs in X18-38 and X18-49 was referred to our previous data (Hanmoto et al. 2003). Refer to results for explanation of letters appearing on chromosomes. Arrows indicate reactivated centromeres.

via chromosomal deletion, or that one or more centromeres become functionally inactivated or suppressed (see Choo 1997). Such centromeres have been designated latent centromeres (Hsu et al. 1975). The mechanisms for inactivation or suppression of active centromeres in dicentrics or multicentrics have yet to be clarified.

With $H$. gracilis, chromosome $1(1 \mathrm{~g})$ is considered to be reconstituted from 3 chromosomes of H. ravenii, chromosome 1 (1r), chromosome 2 (2r) and chromosome 3 (3r), as indicated by comparative analysis of the mitotic prophase (Tanaka 1967) and examination of chromosome configuration in PMCs of $\mathrm{F}_{1}$ hybrids between $H$. gracilis and H. ravenii (Jackson 1962, Ikeda 1987). Jackson (1962) considers the numerical reduction in chromosomes to result from unequal reciprocal translocation and subsequent loss of 2 centromeres.

Neither Jackson (1962) nor Ikeda (1987) could find any unpaired regions in pachytene chromosomes in $F_{1}$ hybrids between the 2 species. Tanaka (1967) noted total chromosome length and distribution of ECRs in mitotic prophase to be basically the same in these species. Accordingly, the consideration of Jackson (1962) that extra centromeres of $H$. ravenii were lost as B chromosomes in chromosomal evolution in this genus would not be valid.

Fig. 2 demonstrates semi-schematically the haploid chromosome complements of 2 rearranged karyotypes with $2 n=5,78-465$ and X18-38 with X18-49, in the mitotic prophase for comparison with the karyotypes of $H$. ravenii and $H$. gracilis. Chromosome length and ECR distribution in 2 aberrant chromosomes in $78-465, \mathrm{~V}_{1}$ and $\mathrm{V}_{2}$, are quite consistent with those in normal $1 \mathrm{~g}$, as is also the case for $\mathrm{V}_{3}$ and " $\mathrm{F}$ " in $\mathrm{X} 18-38$ and $\mathrm{X} 18-49$.

The aberrant chromosomes, $\mathrm{V}_{1}$ and $\mathrm{V}_{2}$ in 78-465, and $\mathrm{V}_{3}$ and " $\mathrm{F}$ " in $\mathrm{X} 18-38$ and $\mathrm{X} 18-49$, would thus appear to be produced by chromosome breakage in $1 \mathrm{~g}$ and additional centromere activity in any given complement may possibly arise from reactivation of either of the 2 latent centromeres at interstitial or subdistal positions in the long arm of $1 \mathrm{~g}$. Chromosome 1 thus shows to have the latent centromeres in the long arm, each possessing the capacity for reactivation.

Increase in the number of centromeres in the chromosome complement induced by centromeric fission has been reported for the metacentric chromosome in rye (Sybenga and de Vries 1987), Hypochoeris radicata (Parker 1987), Vicia faba (Schubert and Rieger 1990), though rarely in humans (Choo 1997), and to produce 2 telocentric chromosomes. There appear to be no reports on in- 
crease in centromeres due to the reactivation of latent centromeres. This paper thus presents for the first time reactivation of latent centromeres.

Additional evidence on latent centromeres should also be obtained using molecular markers such as centromere-specific DNA or proteins (CENPs).

\section{Acknowledgements}

We wish to thank Professors K. Fujikawa and T. Itoh, Kinki University, for their technical assistance in conducting the X-rays irradiation.

\section{References}

Choo, K. H. A., 1997. The centromere. Oxford Univ. Press.

Hanmoto, H., Fujikawa, K., Itoh, T. and Yonezawa, Y. 2003. Repetitious production of similar karyotypes in different plants of Haplopappus gracilis, an annual Asteraceae, following exposure to ionizing radiation. Cytologia. 68: 413-424.

Hsu, T. C., Pathak, S. and Chen, T. R., 1975. The possibility of latent centromeres and proposed nomenclature system for total chromosome and whole arm translocations. Cytogenet. Cell Genet. 15: 41-49.

Ikeda, H. 1987. Cytogenetic studies on the chromosome complements of Haplopappus gracilis $(2 n=4)$ J. Sci. Hiroshima Univ. Ser B, Div. 2, 21: 67-104.

Jackson, R. C. 1957. New low chromosome number for plants. Science. 126: 1115-1116.

— 1959. A study of meiosis in Haplopappus gracilis (Compositae). Amer. J. Bot. 46: 550-554.

- 1962. Interspecific hybridization in Haplopappus and its bearing on chromosome evolution in the Blepharodon section. Amer. J. Bot. 49: 119-132.

- 1973. Chromosomal evolution in Haplopappus gracilis: A centric transposition race. Evolution. 27: 243-256.

Parker, J. S. 1987. Increased chiasma frequency as a result of chromosome rearrangement. Heredity. 58: 87-94.

Schubert, I. and Rieger, R. 1990. Alteration by centric fission of the diploid chromosome number in Vicia faba L. Genetica. 81: $67-69$.

Sybenga, J. and de Vries, J. M. 1987. Variation in orientation of multivalent formed by a reciprocal and a Robertsonian translocation in rye. Biol. Zbl. 106: 409-415.

Tanaka, R. 1967. A comparative karyotype analysis in Haplopappus gracilis $(2 n=4)$ and Haplopappus ravenii $(2 n=8) \mathrm{Cy}-$ tologia. 32: 542-554.

Yonezawa, Y. 1981a. Cytological and cytogenetic studies on the transposition of centromere and the karyotype differentiation in Haplopappus gracilis I. A new-shaped chromosome. Cytologia. 46: 431-441.

- 1981b. Cytological and cytogenetic studies on the transposition of centromere and the karyotype differentiation in Haplopappus gracilis II. Genetic behaviour of the new-shaped chromosome. Cytologia. 46: 443-449. 